\title{
Inclusão da covariância genética direta-materna no modelo para estimar parâmetros e predizer valores genéticos para ganho de peso em bovinos da raça Angus ${ }^{1}$
}

\author{
Luiz Felipe Waihrich Guterres ${ }^{2}$, Paulo Roberto Nogara Rorato ${ }^{3}$, Arione Augusti Boligon 4,5 , \\ Tomás Weber ${ }^{4}$, Jader Silva Lopes ${ }^{4}$, Paulo Rodrigo Santos de Souza ${ }^{2}$ \\ ${ }_{1}^{1}$ Parte da dissertação do primeiro autor apresentada ao Programa de Pós-Graduação em Zootecnia da UFSM, como parte dos requisitos \\ para obtenção do grau de Mestre em Produção Animal. \\ 2 Zootecnista, M.Sc. em Produção Animal. \\ ${ }^{3}$ Departamento de Zootecnia - UFSM. \\ ${ }^{4}$ Curso de Graduação em Zootecnia da UFSM. \\ ${ }^{5}$ Bolsista PIBIC-CNPq.
}

RESUMO - Com o objetivo de estimar o efeito da inclusão da covariância genética direta-materna no modelo de análise sobre as estimativas de parâmetros genéticos e predição de valores genéticos (VG) para ganho médio diário do nascimento à desmama (GMDND) e da desmama ao sobreano (GMDDS), foram utilizados registros de 11.202 e de 4.665 animais da raça Angus, respectivamente, para GMDND e GMDDS. As (co)variâncias foram obtidas adotando-se um modelo animal que, para GMDND, incluiu os efeitos aleatórios genéticos aditivos diretos e maternos e residual, os efeitos fixos de grupo de contemporâneos à desmama, além das covariáveis idade da vaca ao parto (IV) e idade do animal à desmama e, para GMDDS, considerou os efeitos aleatórios descritos e os fixos de grupo de contemporâneos ao sobreano, além das covariáveis IV e idade do animal ao sobreano. Para ambos os modelos, foi considerado aleatório o efeito de ambiente permanente da vaca. As herdabilidades diretas estimadas para GMDND foram 0,25 não considerando e 0,55 considerando a covariância genética direta-materna; comportamento similar foi observado para a herdabilidade materna (0,07 e 0,22). Para GMDDS, as herdabilidades diretas estimadas foram similares: 0,21 não considerando a covariância e 0,22 quando a covariância foi considerada. O teste da razão de verossimilhança mostrou não haver diferença significativa, a 5\% de probabilidade, entre os modelos adotados para ambas as características. As correlações entre os efeitos genéticos aditivos diretos e maternos foram negativas, tanto para GMDND quanto para GMDDS. As correlações de Spearman entre os ordenamentos dos reprodutores, de acordo com seus valores genéticos (rank correlation), obtidos incluindo e não incluindo a covariância entre os efeitos diretos e maternos no modelo de análise (0,88 para GMDND e 0,99 para GMDDS) sugerem que a inclusão da covariância genética direta-materna no modelo é relevante somente para GMDND.

Palavras-chave: correlação de Spearman, efeito materno, ganho médio diário, herdabilidade

\section{Inclusion of genetic direct-maternal covariance in the model to estimate genetic parameters and to predict breeding values for weight gains in Angus cattle}

\footnotetext{
ABSTRACT - This study aimed to evaluate the effect of the inclusion of genetic direct-maternal covariance on genetic parameters estimates and on ranking of breeding values (VG) for average daily gain from birth to weaning age (GMDND) and from weaning to 550 days of age (GMDDS) using records from 11,202 and 4,665 Angus animals respectively. Covariance components were estimated by restricted maximum likelihood method and for GMDND the model included the additive genetic direct, maternal, permanent environmental and residual random effects and the contemporary group at weaning and the covariables age of the cow at calving and age at weaning fixed effects. The model for GMDDS was similar, but included only the contemporary group at 550 days of age and the covariable age at 550 days as fixed effects. Both models included or not (assumed to be null) the genetic direct-maternal covariance. The estimated direct heritabilities for GMDND were 0.55 or 0.25 when the maternal-direct covariance was respectively fitted or not. Respective estimates for maternal heritability were 0.22 and 0.07. The estimated direct heritabilties for GMDDS including or not the genetic direct-maternal covariance were 0.22 and 0.21 respectively. The likelihood ratio test did not indicate significant diference between models for both traits. The Spearman rank correlations between sire VG from both models were 0.88 for GMDND and 0.99 for GMDDS suggesting that the inclusion of the maternal-direct genetic covariance is relevant only for GMDND.
}

Key Words: average daily gain, heritability, maternal effect, Spearman correlation 


\section{Introdução}

Os caracteres utilizados como critérios de seleção são determinados não somente pelo genótipo do animal, mas também pelo efeito da mãe, particularmente no período prédesmama. Conhecer a influência materna sobre os pesos pré e pós-demama e a correlação entre os efeitos genéticos aditivos diretos e maternos é fundamental para obtenção de estimativas de herdabilidade mais precisas e não viesadas (Cabrera et al., 2001).

$\mathrm{O}$ efeito da mãe sobre o bezerro é conseqüência do efeito dos genes da mãe; em relação ao bezerro, este efeito não passa de um efeito ambiental, por isto, pode ser mensurado como apenas parte de todo o efeito ambiental a que o bezerro está sujeito (Perotto, 2003).

Sarmento et al. (2003) afirmaram que o ambiente materno pode influenciar o bezerro tanto na fase pré como pós-natal, sendo que, na fase pós-natal, o manejo dispensado aos animais pode alterar este efeito. Willham (1980) relata que o efeito materno é um dos que contribuem para o valor fenotípico de um indivíduo por parte de sua mãe e deve ser considerado na avaliação genética dos animais, principalmente na fase pré-desmama e que se torna mais intenso com o avançar da idade da fêmea. Todavia, Biffani et al. (1999) relataram evidências do efeito materno também no período pós-desmama. Por outro lado, Willham (1972) afirmou que a habilidade materna, em bovinos de corte, estaria relacionada diretamente à produção de leite da vaca, o que traria reflexos no desempenho do bezerro, principalmente à desmama, embora tenha registrado contribuição do efeito materno também para o peso ao nascer, indicando que outros fatores são importantes na determinação do efeito materno. Similarmente, Meyer et al. (1994) afirmaram que a produção de leite é o principal determinante dos efeitos maternos no crescimento de bezerros das raças de corte.

Entretanto, relatos sobre o antagonismo entre os efeitos genéticos aditivos diretos e maternos foram feitos por Meyer et al. (1994) e Lee \& Pollak (1997), o que, segundo Eler et al. (1989), comprometeria a resposta positiva para potencial de crescimento, sujeito à influência materna. Se a seleção fosse feita com base no valor fenotípico dos animais, uma resposta positiva para potencial de crescimento estaria associada à redução na habilidade materna. Por outro lado, Mercadante \& Lôbo (1997), trabalhando com animais da raça Nelore, não evidenciaram este antagonismo.

Estas diferentes constatações tornam evidente a importância do estudo da inclusão da covariância entre os efeitos genéticos aditivos diretos e maternos nos modelos de análise para que se possa determinar de que forma a inclusão pode influenciar as estimativas de parâmetros genéticos e as predições dos valores genéticos dos animais, uma vez que, em populações, pequenas diferenças nos valores das estimativas podem alterar as posições de um grande número de animais no ordenamento pelo valor genético.

Este trabalho foi realizado com o objetivo de avaliar o efeito da inclusão da covariância entre os efeitos genéticos aditivos diretos e maternos nos modelos de análise sobre a estimativa de coeficientes de herdabilidade e a ordem de classificação dos reprodutores segundo seus valores genéticos.

\section{Material e Métodos}

Foram utilizadas informações de 11.202 e 4.665 registros de desempenho de bovinos da raça Angus para análise do ganho médio diário do nascimento à desmama (GMDND) e do ganho médio diário da desmama ao sobreano (GMDDS), respectivamente. Os dados foram coletados em diversas fazendas localizadas em diferentes regiões do Brasil no período de 1987 a 2001 e foram cedidos pela Gensys Consultores Associados S/C Ltda. e Natura Genética Sul - Americana.

Para aumentar a consistência das estimativas, foram excluídos dos arquivos de trabalho animais provenientes de monta natural, grupos de contemporâneos com menos de dez animais, touros com menos de duas progênies, registros incompletos, animais com GMDND inferior a $333,30 \mathrm{~g}$ ou superior a 1.014,00 g e com GMDDS inferior a $102,70 \mathrm{~g}$ ou superior a $848,30 \mathrm{~g}$. Os limites para exclusão dos dados foram definidos considerando 2,5 desvio-padrão em relação à média, para mais ou para menos.

Foram geradas, usando o pacote estatístico SAS (2001), as seguintes variáveis: GCN (grupo de contemporâneos ao nascimento) - animais nascidos na mesma fazenda, em mesmo ano e estação e pertencentes ao mesmo sexo; GC205 (grupo de contemporâneos ao desmame) - animais nascidos na mesma fazenda, em mesmo ano e estação de nascimento e de desmama e pertencentes ao mesmo sexo e mesmo grupo de manejo à desmama; GC550 (grupo de contemporâneos ao sobreano) - animais nascidos na mesma fazenda, em mesmo ano e estação de nascimento e pertencentes ao mesmo sexo, mesma estação e grupo de manejo à desmama e mesma estação e grupo de manejo ao sobreano; GMDND (ganho médio diário do nascimento ao desmame); GMDDS (ganho médio diário do desmame ao sobreano); estação de nascimento: 1 - animais nascidos nos meses de janeiro a março, 2 - de abril a junho, 3 - de julho a setembro e 4-de outubro a dezembro; estação de desmama: 1 - animais desmamados 
nos meses de janeiro a março, 2 - de abril a junho, 3 - de julho a setembro e 4 - de outubro a dezembro; estação de sobreano: 1 - animais com 550 dias nos meses de janeiro a março, 2 - de abril a junho, 3 - de julho a setembro, e 4 - de outubro a dezembro; P205 - peso ajustado aos 205 dias; e P550 - peso ajustado aos 550 dias. Foram criados dois arquivos de trabalho, um para GMDND e outro para GMDDS.

Para definir as variáveis a serem incluídas nos modelos, foram realizadas análises de variância utilizando-se o pro cedimento PROC GLM (SAS, 2001).

Os componentes de (co)variância utilizados para estimar os coeficientes de herdabilidade direta e materna e predizer os valores genéticos (VG) foram obtidos pelo método da Máxima Verossimilhança Restrita Livre de Derivada, com o programa computacional MTDFREML, descrito por Boldman et al. (2001). Para GMDND, foi adotado um modelo animal considerando aleatórios os efeitos genéticos aditivos diretos e maternos e o residual e, como fixos, os efeitos de grupo de contemporâneos à desmama (GC205), além das covariáveis idade à desmama (ID) e idade da vaca ao parto (IV), efeitos linear e quadrático. Para GMDDS, o modelo animal foi o mesmo, substituindo apenas o efeito de GC205 pelo de grupo de contemporâneos ao sobreano (GC550) e ID por idade ao sobreano (IS). Cada modelo foi utilizado em duas análises, uma considerando a covariância entre os efeitos genéticos diretos e maternos igual a zero e a outra considerando o valor da covariância previamente estimado, diferente de zero. $\mathrm{O}$ efeito aleatório de ambiente permanente da vaca foi incluído no modelo para todas as análises.

Os modelos de análise utilizados podem ser descritos, sob a forma matricial, como:

$$
\mathrm{Y}=\mathrm{X} \beta+\mathrm{Z}_{1} \mathrm{a}+\mathrm{Z}_{2} \mathrm{~m}+\mathrm{Z}_{3} \mathrm{p}+\mathrm{e},
$$

em que: $\mathrm{Y}=$ vetor das observações de cada característica (GMDND e GMDND); X = matriz de incidência associada aos efeitos fixos; $\beta=$ vetor de solução para os efeitos fixos; $\mathrm{Z}_{1}=$ matriz de incidência associada ao efeito genético aditivo direto de cada animal; $\mathrm{a}=$ vetor de soluções para os efeitos genéticos aditivos diretos aleatórios; $Z_{2}=$ matriz de incidência associada ao efeito genético aditivo materno de cada animal; $m$ = vetor de soluções para os efeitos genéticos aditivos maternos aleatórios; $Z_{3}=$ matriz de incidência associada ao efeito de ambiente permanente da vaca; $\mathrm{p}=$ vetor de soluções para os efeitos aleatórios de ambiente permanente da vaca; e = vetor dos resíduos.

O critério de convergência considerado nas análises com o MTDFREML foi de $10^{-6}$, sendo que, a cada convergência, o programa era reiniciado, utilizando-se como valores iniciais as estimativas da análise anterior até o momento em que o resultado do -2 Log não se alterou mais nas últimas três análises.
As matrizes de parentesco estavam constituídas de 15.960 animais para GMDND (arquivo desmama) e por 7.645 animais para GMDDS (arquivo sobreano).

Os modelos foram comparados pelo teste de razão de verossimilhança 1 a $5 \%$ de probabilidade de erro. Segundo Freund \& Walpole (1980), 1 é calculada como a seguir: 1=L2/L1, em que: L2 = valor de máxima verossimilhança do modelo 2 (com a inclusão da covariância aditiva diretamaterna); L1 = valor de máxima verossimilhança do modelo 1 (sem a inclusão da covariância aditiva direta-materna). De acordo com esses autores, para um grande número de observações (n), a distribuição de -2logl aproxima-se geralmente a uma distribuição de qui-quadrado $\left(\mathrm{c}^{2}\right)$ com 1 grau de liberdade, i.e.: $-2 \log 1 \sim \mathfrak{C}^{2}$ an, em que a = nível de significância e $\mathrm{n}=$ grau de liberdade (1). O cálculo de $-2 \log 1$ pode ser simplificado aplicando-se a diferença entre -2 $\log \mathrm{L} 2$ e $-2 \log \mathrm{L} 1$, uma vez que: $1=\mathrm{L} 2 / \mathrm{L} 1=\mathrm{L} 2 \cdot \mathrm{L}^{-1}$, $\mathrm{P} \log 1=\log \mathrm{L} 2$ $-\log \mathrm{L} 1, \mathrm{P}-2 \log 1=(-2 \log \mathrm{L} 2)-(-2 \log \mathrm{L} 1)$. O valor obtido para a estatística do teste da razão de verossimilhança 1 foi comparado ao valor do qui-quadrado tabelado $\left(\mathrm{c}^{2}{ }_{\mathrm{tab}}\right) \mathrm{com}$ um grau de liberdade, considerando $1>\mathfrak{c}_{\mathrm{tab}}=$ efeito significativo.

Os animais foram ordenados pelos seus valores genéticos preditos nas duas análises (com e sem a inclusão da covariância entre os efeitos genéticos diretos e maternos) e a correlação entre os ordenamentos (rank correlation) foi estimada pelo procedimento CORR, opção Spearman, do programa SAS (2001), com o objetivo de avaliar a correspondência entre as classificações dos reprodutores para os diferentes modelos de análise.

\section{Resultados e Discussão}

Os resultados da análise de variância indicaram que, para GMDND, foram significativos os efeitos de GCD $(\mathrm{P}<0,001)$, IV, efeitos linear e quadrático $(\mathrm{P}<0,001)$. Entretanto, esta característica não foi influenciada a 1 e $5 \%$ de significância pela idade à desmama (ID) linear $(\mathrm{P}=0,8419) \mathrm{e}$ quadrática $(\mathrm{P}=0,4421)$; mesmo assim, estes efeitos foram incluídos no modelo para a análise genética; para GMDDS, todos os efeitos incluídos no modelo foram significativos a $1 \%$ (Tabela 1$)$.

Nas Tabelas 2 e 3 são apresentadas as medidas descritivas para as características GMDND e GMDDS, respectivamente.

A média observada para GMDND $(662,63 \mathrm{~g})$ foi superior à relatada por Cardoso et al. (2004) para a raça Angus $(605 \mathrm{~g})$ e inferior à verificada por Cabrera et al. (2001) para a raça Nelore (787 g). A média observada para GMDDS foi de 378,97 g, próxima à descrita por Cardoso et al. (2004) 
para a raça Angus (352 g) e superior à relatada por Cabrera et al. (2001) para a raça Nelore (295 g).

As estimativas dos componentes de (co)variância e dos coeficientes de herdabilidade obtidos para GMDND e GMDDS, em análises univariadas, sem e com a inclusão da covariância entre os efeitos genéticos diretos e maternos e a correlação entre efeitos são apresentadas na Tabela 4.
Os valores estimados para os componentes de variância, para GMDND, foram superiores aos relatados por Malhado et al. (2003), mas, como observado por estes autores, verificaram-se aumentos de 2,4 e 3,4 vezes no valor dos componentes de variância genética aditiva direta e materna quando o efeito da covariância foi incluído no modelo de análise. A covariância negativa entre os efeitos genéticos

Tabela 1 - Análise de variância para ganho médio diário do nascimento à desmama (GMDND) e para ganho médio diário da desmama ao sobreano (GMDDS)

Table 1 - Analyses of variance for average daily gain from birth to weaning (GMDND) and for average daily gain from weaning to 550 days of age (GMDDS)

\begin{tabular}{|c|c|c|c|c|c|c|}
\hline \multirow{2}{*}{$\begin{array}{l}\text { Fonte de variação } \\
\text { Source of variation }\end{array}$} & \multicolumn{3}{|c|}{ GMDND } & \multicolumn{3}{|c|}{ GMDDS } \\
\hline & GL & QM & $\mathrm{P}>\mathrm{F}$ & GL & QM & $\mathrm{P}>\mathrm{F}$ \\
\hline $\begin{array}{l}\text { Grupo de contemporâneos à desmama } \\
\text { Contemporary group at weaning }\end{array}$ & 114 & $490.795,10$ & $<0,0001$ & - & - & - \\
\hline $\begin{array}{l}\text { Grupo de contemporâneos ao sobreano } \\
\text { Contemporary group at } 550 \text { days of age }\end{array}$ & - & - & - & 133 & $469.209,58$ & $<0,0001$ \\
\hline
\end{tabular}

Idade à desmama

Age at weaning

\begin{tabular}{lccc} 
Linear & 1 & 523,21 & 0,8419 \\
$\begin{array}{l}\text { Linear } \\
\text { Quadrática } \\
\text { Quadratic }\end{array}$ & 1 & $7.773,64$ & 0,4421 \\
\hline
\end{tabular}

Idade ao sobreano

550 days of age

\begin{tabular}{|c|c|c|c|c|c|c|}
\hline Linear & - & - & - & 1 & $81.742,44$ & 0,0002 \\
\hline Linear & & & & & & \\
\hline Quadrática & - & - & - & 1 & $83.778,80$ & 0,0001 \\
\hline
\end{tabular}

Idade da vaca ao parto

Age of the cow at parturition

\begin{tabular}{|c|c|c|c|c|c|c|}
\hline Linear & 1 & $2.829 .613,94$ & $<0,0001$ & 1 & $65.146,52$ & 0,0008 \\
\hline Linear & & & & & & \\
\hline Quadrática & 1 & $2.309 .086,09$ & $<0,0001$ & 1 & $63.203,03$ & 0,0009 \\
\hline
\end{tabular}

Quadratic

$\mathrm{GL}=$ graus de liberdade (degrees of freedom); $\mathrm{QM}=$ quadrado médio (mean square); $\mathrm{P}>\mathrm{F}=$ nível de significância (level of significance).

Tabela 2 - Número de observações $(\mathrm{N})$, médias, coeficiente de variação (CV\%), mínimo (Min.) e máximo (Máx.) para ganho médio diário do nascimento à desmama (GMDND, g), idade da vaca ao parto (ID VACA, dias), idade à desmama (ID DESM, dias) e peso à desmama ajustado para 205 dias de idade (P205, kg)

Table 2 - Number of records (N), mean, coefficient of variation (CV\%), minimun (Min), maximun (Max) values for average daily gain from birth to weaning (GMDND, g), age of cow at calving (ID VACA, days), age at weaning (ID DESM, days), and weaning weight adjusted to 205 days of age $(P 205, \mathrm{~kg})$

\begin{tabular}{lcrrrr}
\hline $\begin{array}{l}\text { Característica } \\
\text { Trait }\end{array}$ & $\mathrm{N}$ & $\begin{array}{c}\text { Média } \\
\text { Mean }\end{array}$ & $\mathrm{CV}(\%)$ & $\begin{array}{c}\text { Min. } \\
\text { Min. }\end{array}$ & $\begin{array}{c}\text { Máx. } \\
\text { Max. }\end{array}$ \\
\hline GMDND & 11.202 & 662,63 & 20,94 & 333,30 & $1.014,00$ \\
ID VACA & 11.202 & $1.973,03$ & 37,91 & 743,00 & $5.230,00$ \\
ID SOB & 11.202 & 201,53 & 16,85 & 101,00 & 331,00 \\
P205 & 11.202 & 166,85 & 17,12 & 100,00 & 240,00 \\
\hline
\end{tabular}

Tabela 3 - Número de observações $(\mathrm{N})$, médias, coeficiente de variação (CV\%), mínimo (Mín) e máximo (Máx.) para ganho médio diário da desmama ao sobreano (GMDDS, g), idade da vaca ao parto (ID VACA, dias), idade ao sobreano (ID SOB, dias) e peso ao sobreano (P550, kg)

Table 3 - Number of records (N), mean, coefficient of variation (CV\%), minimun (Min), maximun values (Max) for average daily gain from weaning to 550 days of age (GMDDS, $g$ ), age of cow at parturition (ID VACA, days), age at 550 days (ID SOB, days), and weight at $550 \mathrm{~kg}(P 550, \mathrm{~kg})$

\begin{tabular}{lcrrrr}
\hline $\begin{array}{l}\text { Característica } \\
\text { Trait }\end{array}$ & N & $\begin{array}{c}\text { Média } \\
\text { Mean }\end{array}$ & CV $(\%)$ & $\begin{array}{c}\text { Min. } \\
\text { Min. }\end{array}$ & \multicolumn{1}{c}{$\begin{array}{c}\text { Máx. } \\
\text { Max. }\end{array}$} \\
\hline GMDDS & 4.665 & 378,97 & 40,30 & 102,70 & 848,30 \\
ID VACA & 4.665 & $1.971,74$ & 37,18 & 767,00 & $5.188,00$ \\
ID SOB & 4.665 & 527,99 & 11,05 & 360,00 & 682,60 \\
P550 & 4.665 & 300,52 & 20,18 & 165,30 & 514,60 \\
\hline
\end{tabular}


aditivos diretos e maternos para GMDND corrobora a relatada por Cardoso et al. (2001), de -0,51, e por Malhado et al. (2003), de -0,12.

Os componentes de variância para GMDND aumentaram com a inclusão da covariância no modelo de análise, exceto a variância residual, que representou 65 e $45 \%$ da variância total, respectivamente, sem e com a inclusão da covariância no modelo de análise.

As estimativas de herdabilidade direta obtidas neste estudo (Tabela 4) para GMDND foram de $0,25 \pm 0,03$, sem a inclusão da covariância entre os efeitos direto e materno, e de $0,55 \pm 0,08$, quando incluída a covariância no modelo de análise, sugerindo que sua inclusão altera o valor da estimativa. Comportamento semelhante foi relatado por Meyer et al. (1994), para as raças Hereford e Wokalup na Austrália, e por Albuquerque et al. (1998), para a raça Caracu no Brasil. Coeficientes de herdabilidade para o efeito direto, não considerando a covariância entre os efeitos direto e materno, inferiores aos obtidos neste estudo foram relatados por Cardellino \& Cardellino (1984), para a raça Hereford no Rio Grande do Sul $(0,012 \pm 0,07)$, e por Sarmento et al. (2003), para a raça Nelore na Paraíba $(0,12 \pm 0,04)$. Valores inferiores aos deste trabalho foram relatados por Muniz (2001), citado por Malhado et al. (2003), para a raça Gir $(0,11)$ e Malhado et al. (2003) para a raça Nelore $(0,12)$. Considerando a covariância no modelo de análise, outros autores (Everling et al., 2001; Muniz, 2001, citado por Malhado et al., 2003; Malhado et al., 2003) encontraram valores de herdabilidade para o efeito direto 55,77 e $77 \%$, respectivamente, menores que o encontrado neste trabalho.

Para GMDND, os valores estimados para a herdabilidade materna (Tabela 4) foram $0,07 \pm 00,2$ e 0,22 $\pm 0,04$, sem e com a inclusão da covariância, respectivamente, o que sugere a importância da inclusão do efeito materno no modelo de análise. $\mathrm{O}$ valor estimado sem a inclusão da covariância no modelo foi similar ao encontrado por Malhado et al. (2003) para a raça Nelore $(0,06 \pm 0,02)$ e, quando considerada a covariância, foi similar ao relatado por Sarmento et al. (2003) para a raça Nelore $(0,29 \pm 0,09)$, todavia, foi aproximadamente 77,18 e $27 \%$ maior que os relatados por Marcondes et al. (2000), Malhado et al. (2003), de 18 e 0,05 $\pm 0,01$, respectivamente, ambos para a raça Nelore, e por Cardoso et al. (2001) para a raça Angus $(0,16)$. Ressalta-se que Sarmento et al. (2003) encontraram valores para a herdabilidade materna

Tabela 4 - Componentes de (co)variância e parâmetros genéticos para ganho médio diário do nascimento à desmama (GMDND) e ganho médio diário da desmama ao sobreano (GMDDS)

Table 4 - (Co)variance components and genetic parameters for average daily gain from birth to weaning (GMDND) and average daily gain from weaning to 550 days of age (GMDDS)

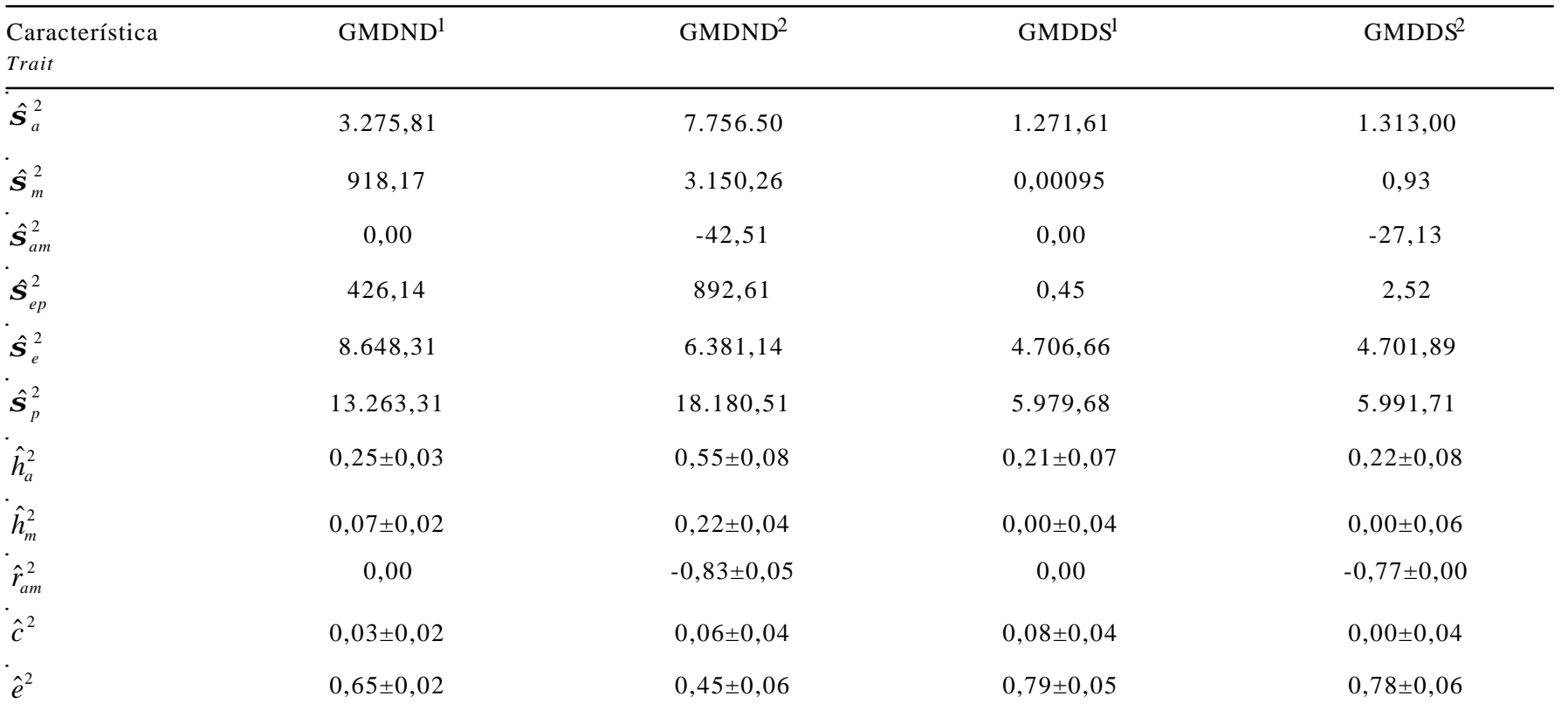

$\hat{\sigma}_{a}^{2}=$ variância genética aditiva direta (direct additive genetic variance); $\hat{\sigma}_{m}^{2}=$ variância genética aditiva materna (maternal additive genetic variance); $\hat{\sigma}_{a m}^{2}=$ covariância entre os efeitos direto e materno (direct-maternalcovariance); $\hat{\sigma}_{e p}^{2}=$ variância de ambiente permanente (permanent environmental variance); $\hat{\sigma}_{e}^{2}=$ variância residual(residual variance); $\hat{\sigma}_{p}^{2}=$ variância fenotípica (phenotypic variance); $\hat{h}_{a}^{2}=$ herdabilidade direta (direct heritability); $\hat{h}_{m}^{2}=$ herdabilidade materna (maternal heritability); $\hat{r}_{a m}^{2}=$ correlação entre os efeitos direto e materno (genetic-maternal effect correlation); $\hat{c}^{2}=$ contribuição do ambiente permanente (permanenteenvironmentalcontribution); $\hat{e}^{2}=$ proporção da variância total devido à variância residual (proportion of the total variance due to residual one).

1 Modelo sem a inclusão da covariância (model not including the direct-maternal covariance).

2 Modelo com a inclusão da covariância (model including the direct-maternal covariance). 
maiores que para a herdabilidade direta, pois espera-se que os genes do bezerro tenham maior influência sobre seu desenvolvimento que os da mãe.

Embora pelo teste de rasão de verossimilhança não tenha havido diferença significativa entre os modelos a 5\% de probabilidade para ambas as características, os resultados obtidos neste trabalho para GMDND parecem sugerir a necessidade da inclusão da covariância entre os efeitos direto e materno nos modelos de análise com o objetivo de obter estimativas mais acuradas.

As estimativas de herdabilidade direta (Tabela 4) para GMDDS foram de 0,21 \pm 0,07 sem a inclusão da covariância e de 0,22 $\pm 0,08$ com a inclusão da covariância no modelo de análise. Esses valores foram próximos aos relatados por Cardoso et al.(2004) para a raça Angus $(0,20)$, porém superiores aos descritos por Wilson et al. (1986) para as raças Angus e Hereford nos Estados Unidos $(0,15$ e 0,16), e por Marcondes et al. (2000) e Malhado et al. (2003), entre 0,08 e 0,11 e de 0,14 $\pm 0,02$ para a raça Nelore.

As correlações genéticas entre os efeitos direto e materno $\left(\mathrm{r}_{\mathrm{am}}\right)$ foram altas e negativas: $-0,83 \pm 0,05$ para o GMDND e -0,77 \pm 0,00 para o GMDDS. Estes resultados foram próximos aos relatados por Sarmento et al. (2003) e similares, quanto ao antagonismo, aos descritos por Marcondes et al. (2000), Cardoso (2001) e Everling et al. (2001), mas, em magnitude, foram superiores aos encontrados por esses autores.

Estes valores de correlações, altas e negativas, apontam antagonismo entre os efeitos dos genes para o potencial de crescimento do bezerro e a habilidade materna, um pouco maior para o GMDND, observando-se que, nesse período, a influência do efeito materno sobre o desenvolvimento do bezerro é maior. Mesmo que resultados de correlações genéticas diretas e maternas negativas sejam comumente encontrados na bibliografia, em modelos nos quais são analisadas outras características de crescimento, como peso ao nascer, peso à desmama e peso ao sobreano (Eler et al., 1995; Robinson, 1996; Meyer, 1997; Ribeiro et al., 2001; Cardoso et al., 2001; Cabrera et al., 2001; Magnabosco et al., 1996), os autores atribuem esses resultados mais à inadequação dos arquivos de análise que propriamente a causas biológicas.

Na comparação entre o ordenamento dos reprodutores realizado sem e com a inclusão da covariância entre os efeitos genéticos direto e materno no modelo de análise, foram estimados coeficientes de correlação (Spearman) iguais a 0,88 para GMDND e a 0,99 para GMDDS, sugerindo que a inclusão da covariância entre os efeitos genéticos direto e materno não altera de forma significativa a classificação dos reprodutores e, portanto, usar ou não a referida correlação no modelo de análise, para GMDDS, não aumenta a acurácia das estimativas. Entretanto, para GMDND, a inclusão da covariância pode promover algum aumento na acurácia das estimativas, visto que $12 \%$ de alteração na ordem de classificação em um população numerosa afetará grande número de animais.

\section{Conclusões}

Ao estimar parâmetros genéticos para a característica ganho de peso médio diário do nascimento à desmama, para a raça Angus, a covariância entre os efeitos genéticos diretos e maternos deve ser incluída no modelo de análise com o objetivo de aumentar a acurácia das estimativas, entretanto, para o ganho de peso da desmama ao sobreano, isso não é necessário.

Embora a diferença entre os modelos não tenha sido significativa a 5\% de significância, pelo teste de razão de verossimilhança, a inclusão da covariância entre os efeitos genéticos direto e materno no modelo de análise altera a ordem de classificação dos animais para a característica ganho de peso do nascimento à desmama e, portanto, é importante sua inclusão no modelo de análise para esta característica.

\section{Literatura Citada}

ALBUQUERQUE, L.G.; QUEIROZ, S.A.; FRIES, L.F. Correlação genética entre produção de leite e crescimento pré-desmame em bovinos da raça Caracu. In: REUNIÃO ANUAL DA SOCIEDADE BRASILEIRA DE ZOOTECNIA, 35., 1998, Botucatu. Anais... Botucatu: Sociedade Brasileira de Zootecnia, 1998. (CD-ROM)

BIFFANI, S.; MARTINS FILHO, R.; MARTINI, A. et al. Fatores ambientais e genéticos sobre o crescimento ao ano e ao sobreano de bovinos Nelore, criados no Nordeste do Brasil. Revista Brasileira Zootecnia, v.28, n.4, p.468-473, 1999.

BOLDMAN, K.H.; KREISE, L.A.; Van VLECK, L.D. et al. A manual for use for MTDFREML. A set of programs to obtain estimates of variance and covariances (DRAFT). Lincoln: Departament of Agriculture/Agricultural Research Service, 2001. 120p.

CABRERA, M.E.; GARNERO, A. del V., LÔBO, R.B. et al. Efecto de la incorporación de la covarianza genética directa-materna en el análisis de características de crescimiento em la raza Nelore. Livestock Research for Rural Development, v.13, n.3, p.1-6, 2001.

CARDELINO, R.A.; CARDELlinO, M.G.V. Herdabilidades dos caracteres de produção à desmama em bovinos Hereford no Rio Grande do Sul. Revista Brasileira Zootecnia, v.13, n.4 p.557-563, 1984.

CARDOSO, F.F.; CARDELINO, R.A.; CAMPOS, L.T. Componentes de (co)variância e Parâmetros genéticos de características pósdesmama em bovinos da raça Angus. Revista Brasileira Zootecnia, v.33, n.2, p.313-319, 2004

CARDOSO, F.F.; CARDELlinO, R.A.; CAMPOS, L.T. Componentes de (co) variância e parâmetros genéticos parra 
caracteres produtivos à desmama de bezerros Angus criados no estado do Rio Grande do Sul. Revista Brasileira Zootecnia, v.30, n.1, p.41-48, 2001.

ELER, J.P.; LÔBO, R.B.; DUARTE, F.A. Avaliação dos efeitos genéticos direto e materno em pesos de bovinos da raça Nelore criados no estado de São Paulo. Revista Brasileira Zootecnia, v.18, n.2, p.112-123, 1989.

ELER, J.P.; van VLECK, L.D.; FERRAZ, J.B.S. et al. Estimation of variances due to direct and maternal effects for growth traits of Nelore cattle. Journal of Animal Science, v.73, n.11, p.3253-3258, 1995.

EVERLING, D.M.; FERREIRA, G.B.B.; RORATO, P.R.N. et al. Estimativas de herdabilidade e correlação genética para características de crescimento na fase de pré-desmama e medidas de perímetro escrotal ao sobreano em bovinos Angus-Nelore. Revista Brasileira Zootecnia,v.30, n.6, p.2002-2008, 2001 (supl. 1).

FREUND, J.F.E.; WALPOLE, R.E. Mathematical statistics . 3.ed. New Jersey: Prentice-Hall, 1980. 548p.

LEE, C.; POLLAK, E.J. Relationship between sire x year interactions and direct-maternal genetic correlation for weaning weight of Simental cattle. Journal of Animal Science, v.75, n.1, p.68-75, 1997.

MAGNABOSCO, C.U.; FAMULA, T.R.; LOBO, R.B. et al. Estimativas de parâmetros genéticos e de ambiente de características de crescimento em bovinos da raça Nelore. In: REUNIÃO ANUAL DA SOCIEDADE BRASILEIRA DE ZOOTECNIA, 33., 1996, Fortaleza. Anais... Fortaleza: Sociedade Brasileira de Zootecnia, p.142-144, 1996.

MALHADO, C.H.; MARTINS FILHO, R.; LÔBO, R.B. et al. Efeito da inclusão da covariância entre os efeitos direto e materno sobre os parâmetros genéticos de ganho de peso pré-desmama e pós-desmama. In: REUNIÃO ANUAL DA SOCIEDADE BRASILEIRA DE ZOOTECNIA, Santa Maria. Anais... Santa Maria: Sociedade Brasileira de Zootecnia, 2003. (CD-ROM)

MARCONDES, C.R.; BERGMANN, J.A.G.; ELER, J.P. et al. Análise de alguns critérios de seleção para características de crescimento na raça Nelore. Arquivo Brasileiro de Medicina Veterinária e Zootecnia, v.52, n.1, p.83-89, 2000.

MERCADANTE, M.E.Z.; LÔBO, R.B. Estimativas de (co)variância e parâmetros genéticos e de ambiente de característica de crescimento de fêmeas de um rebanho Nelore. Revista Brasileira Zootecnia, v.26, n.6, p.1124-1133, 1997.

MEYER, K. Estimates of genetic parameters for weaning weight of beef cattle accounting for direct-maternal enviromental covariances. Livestock Production Science, v.52, n.1, p.187 -199, 1997.

MEYER, K.; CARRIK, M.J.; DONNELLY, B.J.P. Genetic parameters for milk production for australian beef cows.and weaning weight of their calves. Journal of Animal Science, v.72, n.5 p.1155-1165, 1994.

PEROTTO, D. O efeito materno no melhoramento de gado de corte. In: CURSO DE MELHORAMENTO GENÉTICO DE GADO DE CORTE, 2003, Campo Grande. Anais... Campo Grande: Embrapa-Geneplus, 2003. p.53-80.

RIBEIRO, M.N.; PIMENTA FILHO, E.C.; MARTINS, G.A. et al. Herdabilidade para efeitos direto e materno de características de crescimento de bovinos Nelore no estado da Paraíba. Revista Brasileira Zootecnia, v.30, n.4, p.1224-122, 2001.

ROBINSON, D.L. Estimation and interpretation of direct and maternal genetic parameters for weights os australian Angus cattle. Livestock Production Science, v.45, n.1, p.1-11, 1996.

SARMENTO, J.R.L.; PIMENTA FILHO, E.; RIBEIRO, M.N. et al. Efeitos ambientais e genéticos sobre o ganho em peso diário de bovinos nelore no estado da Paraíba. Revista Brasileira de Zootecnia, v.32, n.2, p.325-330, 2003.

STATISTICAL ANALYSIS SYSTEMS - SAS. User's guide . Version 8. 1.ed. Cary: 2001. 956p.

WILLHAM, R.L. The role of maternal effect in animal breeding: III. Biometrical aspects of maternal effects in animals. Journal of Animal Science, v.35, n.6, p.1288-1302, 1972.

WILlHAM, R.L. Problems in estimating maternal effects. Livestock Production Science, v.7, n.5, p.405-418, 1980.

WILSON, D.E.; BERGER, P.J.; WILLHAN, R.L. Estimates of beef growth trait variances and heritabilities determined from field records. Journal of Animal Science, v.63, n.2, p.386-394, 1986. 